\title{
Studies on Online Publication and International Development for Academic Journals
}

\section{Chanti Wu}

\author{
Ningbo Dahongying University, Ningbo City, Zhejiang Province 315175
}

\begin{abstract}
Development of academic periodicals has long been focused on by academic and journal communities. Having consulted some researches and related materials about online publication for domestic academic journals, certain cognition has been gained about current situations and future development at home and abroad of academic journals' online publication. Therefore, research and development scope of academic journals' online publication will be widen, international awareness of academic journals' online publication and international exchange on academic journals will be intensified to create international exchange platform which is an significant channel to propel academic journals forward international community. At the same time, brand establishment on China's academic journals, as a new basis for its intentional development, helps the internationalization of academic journals at a rapid speed. As a result, building international academic periodicals in full efforts plays a significant role in comprehensive development on china's academic journals.
\end{abstract}

Keywords: Academic journals; Online publication; International awareness; Brand creation

\section{学术期刊网络出版及国际化发展研究}

\author{
伍婵提 \\ (宁波大红鹰学院，浙江 宁波 315175)
}

摘要:关于学术期刊的未来发展, 一直是学术界和期刊界关注的一个重点。通过对国内学术期刊网络出版的研究及相关 资料的查阅, 对于学术期刊网络出版的发展现状以及未来国内外发展有了一定的认知。提高学术期刊网络出版的研发领域、 强化学术期刊网络出版的国际化意识、加强国内外学术期刊的交流、创建国际化交流平台, 是推动学术期刊走向国际化的重 要途径。同时构建中国学术期刊的品牌化也是学术期刊国际化发展的新基点, 品牌创建有助于快速促进学术期刊的国际化。 全力打造国际化学术期刊, 对于促进中国学术期刊的整体发展具有重要意义。

关键字: 学术期刊; 网络出版; 国际化意识; 品牌创建

中图分类号: G237 文献标志码: A

\section{1 学术期刊网络出版研究现状}

\section{1 国外研究现状}

随着世界科研的进步，网络出版已经成为国际科研教育理论交流的一种手段。对于网络出版的未来发 展方向以及进一步完善网络出版途径, 提高网络出版水平等各方面, 国内外学者都进行了不同程度的研究。 
由于国外的网络发展环境, 国外学者对于网络出版的研究已经深入。1990 年 Sharon Rogers 和 Charlene Hurt 提出了建立一个跨学科的学术交流系统用于网络电子期刊的出版。1999 年 John W. T Smith 认为学术 期刊网络出版应该建立一个主题焦点, 并提出期刊的分解出版模式。实现了传统期刊与网络期刊的交叉互 补性发展, 并且使得期刊的学术观点争鸣越加充分。近年来国外学者先后从技术、标准、管理、出版模式 等多方面对于学术期刊的网络出版进行了探索研究。2003 年 BOAL 提出了建立自我存档的电子档案和开放 存取期刊两种途径以实现学术期刊网络出版的开放存取版模式。伴随着网络科技的进步, 国外的学术期刊 网络出版在技术以及市场、管理模式上都形成了一定的规模与制度。

\section{2 国内研究现状}

随着我国网络技术的发展, 网络出版在期刊出版市场上的发展越发耀眼。学术期刊的网络出版作为期 刊经营的一种发展渠道, 起初在我国的发展并不顺利, 网络出版的版权问题、网络出版市场的低保证以及 网络出版市场的低传播率使得网络出版面临众多问题。2001 年我国修订的《中华人民共和国著作法》确立 了网络出版的传播权, 并在 2006 年确立了版权保护政策, 为学术期刊的网络发展提供了良好的环境。

面对学术期刊的网络出版发展, 我国的研究学者一致认为网络出版在促进学术交流方面具有一定的现 实发展意义。在这一认知下, 我国研究学者对于如恶化发展网络出版进行了探索研究。李红教授建议我国 以国家力量支配、整合、挖掘期刊出版资源, 建立一个精品的学术期刊网络出版平台, 并搭建相应的支撑 体系。赵欧和李宁教授则在合理注意义务方面指出了网络出版发展的问题, 并指出拥有网络出版许可的机 构应该在著作权、链接、网络广告、科技保密、政治问题等方面做到义务并且有效的处理。

\section{2 学术期刊网络出版发展现状}

学术期刊一直是国内期刊发展的一个侧重点, 但相对于其他期刊的发展, 学术期刊的发展明显狭窄。 学术期刊的网络出版发展不仅仅受限于专业人员的自身学术水平和思想, 同时也受限于阅读市场以及国内 出版的发行力度和传统出版市场份额。

\section{1 传统市场份额位居主导}

传统出版作为学术期刊发表的一个重要途径, 它的发展以及市场控制能力, 对于学术期刊的市场具有 重要作用。在传统意义上学术期刊的发表一般依赖于传统的期刊出版, 学术期刊对于传统期刊出版的看重 明显比大于网络出版。虽然学术期刊在传统出版上相对于其他期刊一直存在劣势, 但由于起初的思想定位 更多的专家学者更愿意通过传统方式出版刊物。

虽然网络出版给传统期刊出版带来了一定的冲击, 但传统期刊在期刊市场上仍有很大的潜力。从近五 年的数据调研报告来看, 图书出版整体上是增长的, 且纸介质出版的品种、出版总印张、总印数、总定价 都保持小幅度增长。我国的图书出版数以及新书出版类型和总数相对往年出版虽有浮动, 但却也保持向上 增长。这说明传统出版在与网络出版的对抗中仍有一定的优势。同时由于国内网络出版的发展并不成熟、 渠道上的低保障, 导致专家学者在选择投稿途径上更偏向传统出版。这也使得传统出版在学术期刊发表上 
保留了一定的市场优势。

\section{2 网络出版思想限制}

虽然网络出版的发展在国内外市场上有了一定的基础和地位，网络出版和传统出版两者的发展各有优 劣, 但是网络化的出版在思维上限制了一定的市场。随着互联网的发展, 网络已经成为我们生活中必不可 少的一部分, 但是互联网的人群更多的锁定在中青年以及未成年上的身上。由于老年人在学习以及接触能 力上的差异, 以及对传统教育思想的充分肯定, 致使部分老年学术专家、学者在网络出版上产生了思想限 制。

网络出版对于学术期刊的要求在一定程度上更为严格, 因为网络出版的受众在一定程度上明显大于传 统期刊的受众。同时在国内外的期刊交流上, 更多的交流依赖于网络期刊。由于国际交流的需要, 网络期 刊在发表上对于期刊内容的审核要求更为严格, 但是由于部分专业人员在内容上缺乏一定的专业性以及观 点的说服力上面临退稿, 这使得专家、学者在自身专业水平限制和网络审核要求严格的基础上, 对于网络 出版形成了一定的思维行为规避。

\section{3 学术期刊网络出版发展趋势}

\section{1 构建合理投稿渠道}

随着网络科技的发展, 互联网时代的到来, 网络出版已经成为国内外期刊出版的一条重要途径。传统 期刊的发展虽然保持一定的基础, 但是在数字化出版的大众化潮流下, 传统期刊的转型已经成为一种社会 发展的必然结果。在传统期刊转型的过程中, 网络出版的发展也必须保持一定的发展进度, 从而弥补网络 出版自身的劣势。

在我国的网络出版上，我们很容易发现其存在的渠道问题。网络出版首先要具有良好的投稿渠道，然 后才能进一步的发展。一本期刊的出版与发表离不开投稿人员的支持, 稳定的稿件来源与接收渠道对于网 络出版而言是最为关键且最为基础的发展条件。因此构建合理而稳定的投稿渠道对于网络出版至关重要。 在网络科技的时代下, 构建网络出版渠道依靠的还是互联网络, 因此学术期刊的网络出版首先要构建网络 出版平台, 为投稿人提供一个稳定、有保证的网络渠道。目前我国网络出版渠道发展并不成熟, 这也就使 得构建一个合理而稳定且有保证的网络出版渠道成为未来网络出版的一个新开拓点。

\section{2 打造专业化品牌专线}

目前我国的网络出版市场是在逐渐发展的阶段, 相对于传统学术期刊出版市场, 数字化的网络出版能 更方便、更迅速的走进国家级发展平台。数字化网络的出版对于市场的快速发展具有一定的时效性与便利 性。面对现如今我国相对纷杂的学术期刊网络出版平台, 打造专业化学术期刊网络平台对于快速树立学术 期刊网络出版专业品牌至为重要。

打造我国专业化的学术期刊平台, 建立知名的学术期刊品牌是大数据时代下学术期刊发展的新机遇。 相对于国外知名的学术期刊网络出版平台, 我国的学术期刊发展迟缓且知名度低。目前我国较大的学术期 
刊网络出版平台有中国学术期刊网、中国知网、万方数据平台、维普网, 在这四大平台中中国知网以含括 面积广、发展较为完善而拥有一定的国内外发展优势。在这样的一个市场环境内, 打造一个全新的专业化 网站需要一定的时间与精力, 相对而言利用我国现有的资源大力支持我国目前市场内的知名网站鼓励其对 外国际化发展是快捷而有效的。因此在网络出版的未来发展市场上, 打造专业化的品牌出版平台将是推进 网络出版发展的重要举措。

\section{3 网络与传统协同发展}

在数字化网络出版的快速发展下, 传统出版虽面临挑战, 但是其原有的市场基础以及国内外教育的需 求使得传统出版存在一定的发展优势。因此在未来的市场中网络出版与传统出版并非一定要一争高下, 也 可以携手并进。2 014 年习近平主席提出了 “协同发展” 的观念, 2015 年李克强总理提出了 “互联网+” 的 概念, 两者都强调了融合共赢的发展理念。随着国家政策的提出以及发展新观念的快速应用, 网络出版与 传统出版的融合发展也必将成为未来出版市场的新方向。

在已有的文献分析中发现, 现有的 “互联网+” 更多的是对新闻出版业和图书出版业的研究与引用, 对于学术期刊的 “互联网+” 研究却还没有受到重视。传统出版业的转型与创新已经成为传统出版业发展 的重要一步, 在这一前提下学术期刊的网络出版必须抓紧机遇, 强化与传统学术出版的合作, 加快学术期 刊的发展步伐, 提高学术期刊的市场地位。只有在保证一定的市场基础的前提下, 学术期刊的网络出版才 能加快国际化进程。

\section{4 学术期刊网络出版国际化发展路径}

\section{1 提升出版方国际化意识}

学术期刊作为国内外学术交流的一个传播媒介, 对于学术的传播与交流至关重要。我国的学术期刊要 想达到一定的学术高度, 除了加强对学术研究人员的自身水平培养之外, 同时也必须提升我国学术期刊出 版方的国际化发展意识。国际化的发展并不是单方面的要求, 一本优秀的学术期刊除了学术研究人员的自 身努力, 还需要学术出版方对于学术文章的把控度, 以及对学术期刊发展方向的引领。

网络出版相对于传统出版的差别一定程度上在于传统出版对于文章的要求以及审核更为严格, 而网络 出版对于文章的审核存在一定的缺陷, 其注重的是一些格式及排版等问题而非内容的科研性。因此提升网 络出版方的国际化意识, 强化网络出版方对于出版期刊的审核, 是提升学术期刊质量, 打开学术期刊网络 出版国际化的前提。同时网络期刊出版方作为我国学术期刊国际化发展的一个纽带, 必须时刻关注学术的 国际化研讨, 随时掌握国际化学术发展的方向。学术期刊的自身质量与价值是其走向国际的关键, 同样网 络出版方对于学术期刊的质量把控也非常重要, 故提升出版方的国际化意识是促进网络出版国际化发展的 思想基础。

\section{2 拓展研究领域提升研究品质}

学术期刊的质量水平是衡量其是否具有走进国际化交流平台实力的一个重要准则, 一定的学术研究意 
义以及科研发展创新是走进国际化的必备特征。拓展学术研究领域一方面依靠学术研究者自身知识的积累 与学习, 另一方面依靠网络期刊管理部门的支持与引导。

首先国家层面上应当给予科研创新人员一定的支持, 对于科研创新性学术论文给予一定的优先发表鼓 励, 为学术论文开拓良好的发展空间。其次网络期刊管理部门和出版编辑部门应当积极引进国外优秀期刊 论文, 打开学术专家、学者的眼界以及知识层面。鼓励期刊编辑社聘请国内外知名学者专家坐镇, 保证期 刊内容的学术性, 提高学术期刊的时效性, 以及调控和指导学术研究领域及未来发展方向。学术领域的提 升离不开学术专家、学者在研究领域的眼界及对学术知识的积累和对社会发展的敏感度, 这就需要学术专 家、学者在自身学习的基础上加大对社会的探索与研究。学术专家及学者应以团体形式, 加强学术界的交 流与探索, 完善学术界的科研理论, 从自身以及团体建设两方面提升学术界的研究领域。

\section{3 重视合作强化传播}

中国的学术研究要想打开国际的大门, 必须要坚持 “引进来, 走出去” 的发展策略。首先国内的网络 出版要采取开放式的策略, 完全打开国内国际学术交流的大门。大力邀请国外学术期刊专家、学者的稿件, 积极举办国内外学术研讨会议及活动, 引进国际著名期刊在国内的出版发行。注重与国外出版编辑及网络 出版的合作, 坚持国际期刊标准, 聘请国际知名学者加入评审团, 平衡国内期刊编辑团队的海外比例, 完 善合作交流平台。

学术期刊网络出版的国际化发展一方面要依靠国内外的合作与交流, 另一方面还需要加强对国外市场 的营销宣传。国内的学术期刊要走向国际化一是要注重其学术期刊的质量, 二是强化营销与传播。只有在 国际上拥有一定的市场, 国内的网络期刊出版才能快速获得发展。网络的发展正是网络期刊出版的一个优 势, 学术期刊网络出版可以在网络上建立一个专门的海外发行站点和海外营销传播团队, 或者与世界大型 期刊宣传点进行合作，扩大国内学术期刊网络出版的阅读群体，提升其国际影响力与知名度。

\section{5 结语}

我国作为一个期刊发行大国, 在国际上发行的期刊数量以及文章被引用比例近年来居高不下, 但是在 这样的一个情况下, 我国学术期刊的国际影响力仍未得到一个提升。这使得我国学术研究人员以及科研人 员在未来学术期刊的发展上加深了创新及研究领域, 并对于我国未来的学术期刊投入了更多精力。相信在 未来的国际市场上我国的学术期刊一定会大放异彩。学术期刊的网络出版作为时代发展的产物, 在国际网 络发展的时代下拥有明显的优势。目前我国一部分学术期刊网络出版已经走上国际平台, 相信在不久的将 来会有更多的学术期刊网络出版出现在国际化平台上。

\section{6 致谢}

【基金】教育部 2012 年规划课题 “学术期刊网络出版的国际化路径和前景研究” 阶段性成果 (课题 编号: 12YJA860033); 宁波市哲学社会科学学科带头人培育项目阶段性成果 (课题编号:G15-XK21) 


\section{Acknowledgments:}

This work was supported by project of Ministry of Education of China(Grant No.12YJA860033) named Research on Internationalization and Prospect of Network Publishing of Academic Journals and project of Cultivation of Ningbo Philosophy and Social Science Leaders (Grant No.G15-XK21).

\section{参考文献}

[1] 李红, 中国精品学术期刊数字出版平台刍议 [J]。图书情报工作, 2009（16）：145-148, 73.

[2] 赵鸥, 李宁. 科技期刊网络出版者应尽的合理注意义务一以江苏省科技期刊网络出版现状为例 [J]. 编辑学报,

[3] 余树华. 论期刊数字化与学术期刊体制创新 $[J]$. 出版发行研究, 2012（10）:5-6.

[4] 陈颖, 陈玉霞. 传统期刊的数字化转型路径 [J]. 四川师范大学 学报 (社会科学版), 2012, 39（ 5) : $148-149$.

[5] 邹波, 杭丽芳. 学术期刊优先出版的现状、问题与建议 $[J]$. 出版发行研究, 2015 ( 8) :63.

[6] 刘英. 我国学术期刊网络出版研究综述 $[J]$. 河海大学 学报（哲学社会科学版），2007（4）

[7] 中国学术期刊国际引证报告：2012[R]. 北京：中国学术 期刊 (光盘版) 电子杂志社, 2012 .

\section{References:}

[1] Li Hong. Digital Publishing Platform of Chinese Academic Journals [J].Library and Information Service, 2009(16):145-148, 73. 2011, 23(2): 148-150.

[2] Zhao Ou, Li Ning. Reasonable Obligations of Web Publishers of Sci-tech Periodicals -Jiangsu Province Science and Technology Journal Network Publication Status as an Example [J].Acta Editologica, 2011, 23(2):148-150.

[3] Yu Shuhua. On Digitization of Periodicals and the Innovation of Academic Journals. Publishing Research, 2012 (10): :5-6.

[4] Chen Ying, Chen Yuxia. Digital Transformative Channel of Traditional periodicals [J].Journal of Sichuan Normal University (Social Science Edition), 2012, 39(5): 148-149.

[5] Zou Bo, Hang Lifang. Current Status, Problems and Suggestions on Privileged Publishing of Academic Journals [J].Publishing Research, 2015 (8): 63.

[6] Liu Ying. A Review of the Research on Network Publication of Academic Journals in China [J].Journal of Haihe University( Philosophy and Social Science Edition),2007(4)

[7] International Citation Report of China’s Academic journal: 2012 [R].Beijing: China’s Academic Periodicals (CD Edition) E-magazine Press, 2012. 